\title{
Sirevirus LTR retrotransposons: phylogenetic misconceptions in the plant world
}

\author{
Alexandros Bousios ${ }^{1 *}$ and Nikos Darzentas ${ }^{1,2}$
}

\begin{abstract}
Sireviruses are an ancient and plant-specific LTR retrotransposon genus. They possess a unique genome structure that is characterized by a plethora of highly conserved sequence motifs in key domains of the non-coding genome, and often, by the presence of an envelope-like gene. Recently, their crucial role in the organization of the maize genome, where Sireviruses occupy approximately $21 \%$ of its nuclear content, was revealed, followed by an analysis of their distribution across the plant kingdom. It is now suggested that Sireviruses have been a major mediator of the evolution of many plant genomes. However, the name 'Sirevirus' has caused confusion in the scientific community in regards to their classification within the LTR retrotransposon order and their relationship with viruses - a situation that is not unique to Sireviruses, but also affects other LTR retrotransposon genera. Here, we clarify the phylogenetic position of Sireviruses as typical LTR retrotransposons of the Copia superfamily and explain that the confusion stems from the discrepancy in the categorization of LTR retrotransposons by the two main classification systems: the International Committee on the Taxonomy of Viruses (ICTV) system and the unified classification system for eukaryotic transposable elements. While the name 'Sirevirus' has been given by ICTV, we show that the transposable element system, which is more suitable for eukaryotic genome studies, lacks an appropriate taxonomic level for describing them. We urge for this inconsistency to be addressed. Finally, we provide data suggesting that of the three ICTV-proposed genera of the Pseudoviridae (that is, Copia) family, only Sireviruses form a monophyletic group, while the phylogenetic distinction between Pseudoviruses and Hemiviruses is unclear. We conclude that because of their ongoing important contribution to the classification of transposable elements, these schemes need to be frequently revisited and revised - as shown by the example of the Sirevirus LTR retrotransposon genus.
\end{abstract}

Keywords: Sirevirus, Transposable elements, LTR retrotransposons, ICTV, Phylogenetic analysis, Pseudoviridae, Copia, Plant genomes, Maize

\section{Background}

There are two main classification systems that include LTR retrotransposons (LTR-RTNs) in their taxonomies: (1) the International Committee on the Taxonomy of Viruses (ICTV), which categorizes the plethora of viruses into a single scheme that reflects their evolutionary relationships [1]; and (2) the unified classification system for eukaryotic transposable elements (TEs), which was proposed in a seminal 2007 Nature Review paper [2], and provides standardized nomenclature rules and simple classification strategies for the efficient identification of

\footnotetext{
*Correspondence: alexandros.bousios@gmail.com

'Institute of Applied Biosciences, Centre for Research and Technology Hellas, Thessaloniki 57001, Greece

Full list of author information is available at the end of the article
}

eukaryotic TEs - these include the 80-80-80 rule, which allocates in the same family TEs of minimum length of 80 bp with $>80 \%$ sequence similarity in $>80 \%$ of the length of their coding/internal domain, or of their terminal repeat regions, or both. Other available classification systems such as the Springer Index of Viruses [3], the ITIS Catalogue of life [4], and the Description of Plant Viruses (DPV) index [5] use the same nomenclature as ICTV (see below).

Based on structural and coding sequence similarities of their genomes, LTR-RTNs are evolutionarily related to retroviruses. The most plausible scenario suggests that retroviruses evolved from Gypsy LTR-RTNs after the acquisition of an envelope gene [6], which permitted an infectious extracellular stage. Due to this relationship,

\section{Biomed Central}


and although a limited number of LTR-RTNs contain a putative envelope-like gene, of which only the Gypsy element in Drosophila has been found to be infectious [7], ICTV has incorporated LTR-RTNs in its virus-based scheme and classified them as Pseudoviridae (that is, Copia) and Metaviridae (that is, Gypsy). Both families are divided in three genera: for Pseudoviridae these are the Sirevirus, Pseudovirus, and Hemivirus genera.

This article focuses on Sireviruses and their position within the virus- and TE-based classification systems. We highlight an inconsistency where only ICTV, the less appropriate system for classifying LTR-RTNs, includes a description for the taxonomic level that corresponds to Sireviruses. We argue that this taxonomic gap in the TEbased system should be rectified, as it currently confuses the scientific community, especially in genome annotation studies where ICTV is not broadly used. Finally, we indicate that only Sireviruses form a monophyletic group within the Pseudoviridae and that the phylogenetic basis for the division of Pseudoviruses and Hemiviruses is unclear.

\section{Discussion}

\section{Research on Sireviruses}

Sireviruses are an ancient LTR-RTN genus, and the only one that has exclusively proliferated within the plant kingdom. Their position within the evolutionary history of LTR-RTNs across the eukaryotic tree of life, and how they emerged in the flowering plant lineage, has been beautifully depicted by Llorens et al. [8]. It is the only Pseudoviridae genus whose members may contain a putative envelope-like gene [9]. Due to their host preference they were originally termed Agroviruses [10], before being renamed to Sireviruses by ICTV. Single Sirevirus elements such as SIRE1 [11,12], Opie, and $J i$ $[13,14]$ have been extensively studied; however, with the exception of SIRE1, the Sirevirus origin of most of these has often been neglected. There have also been a limited number of studies that have collectively analyzed Sireviruses on the genus level $[9,10,15,16]$, or correctly annotated as such the Sirevirus part (or other ICTV-derived LTR-RTN genera) of the TE complements of sequenced genomes $[17,18]$.

Recently, a series of publications from our group shed new light on this LTR-RTN genus and made it possible to properly uncover and discuss their integrative impact on their host genomes. Their unique structure among LTR-RTNs was initially revealed, characterized by a multitude of highly conserved sequence motifs within the extremely divergent non-coding part of their genome [19]. An algorithm for their accurate identification was then developed [20], followed by the elucidation of their crucial role in the organization and evolution of the maize genome, of which Sireviruses occupy approximately $21 \%$ and $90 \%$ of the Copia population [21]. Most recently, the MASiVEdb database was released that catalogues their distribution and abundance across a wide range of plant hosts [22] (http://bat.infspire.org/databases/masivedb/). Overall, it is now hypothesized that the amplification/removal cycles of Sireviruses may have been an important factor in the evolution and current make-up of many plant genomes.

\section{Taxonomic inconsistencies and classification suggestions}

There exists uncertainty in the scientific community, in particular non-virologist experts, in regards to the names of the ICTV-derived LTR-RTN genera. Possibly due to their 'virus' suffix, scientists are misled to believe that Sireviruses (and the other Pseudoviridae and Metaviridae genera) are viral species and not typical LTR-RTNs.

The confusion is compounded by the discrepancy in the categorization of LTR-RTNs by the two main classification systems, of which only ICTV provides a taxonomic level ('genera') between 'families' and 'species' (or 'superfamilies' and 'families', respectively, in the TE-based system) (Figure 1A). Each of the three Pseudoviridae and Metaviridae genera contain a few representative species, which in the majority of cases are known LTRRTNs. For instance, the name 'Sirevirus' was derived from the well-studied SIRE1 element of the soybean genome [11]. In contrast, the TE-based system is devoid of a similar taxonomic level, resulting in a phylogenetic 'jump' from superfamily (for example, Copia) to family (for example, SIRE1) (Figure 1A).

Perhaps, the reason for this omission was the difficulty in correctly assigning LTR-RTN families into genera. However, in the case of Sireviruses we have shown that both gene-derived phylogenetic analysis and genome characteristics can reliably distinguish Sirevirus elements $[19,21,22]$. Hence, considering that the TE-based system is the preferred and more suitable scheme for eukaryotic genome studies, this intermediate taxonomic level between superfamilies and families has now become feasible and meaningful.

On the other hand, it is important that LTR-RTNs should remain within the ICTV classification system, even though they are not true viruses. Their evolutionary relationship to retroviruses (Retroviridae family) is a longlasting puzzle that can only be efficiently addressed within the ICTV taxonomy. Aided by the accumulation of new retroviruses and LTR-RTN elements from sequencing projects across the tree of life, it will soon become possible to better understand their phylogenetic pedigree.

\section{Phylogenetic relationships within the ICTV Pseudoviridae family}

The assignment of individual elements into the three Pseudoviridae genera is a difficult task, due to significant 


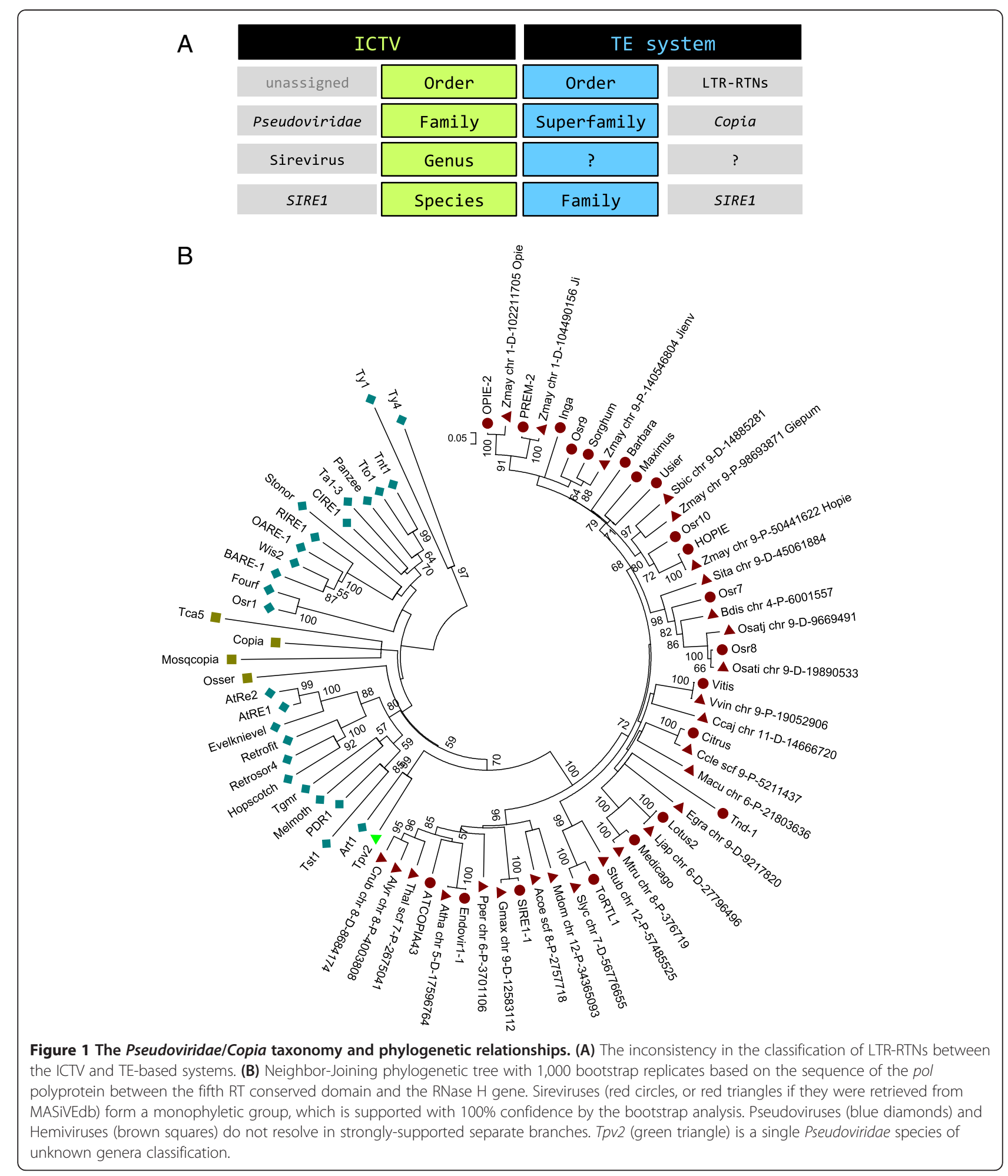

variances in similarity between the genes of their gag-pol domain [1]. The initial criterion used in ICTV was the length of the tail of the tRNA molecule that is used as a primer to initiate reverse transcription. Hemiviruses use only a short segment of tRNA in comparison to Pseudoviruses, with no available information for Sireviruses. NeighborJoining (NJ) phylogenetic analysis based on the reverse transcriptase (RT) gene was then employed for the between- and within-genus separation into distinct species. As a result, and although the properties of their preferred 
tRNA molecule were not known, Sireviruses were considered a separate genus, as its representative species were the only ones that consistently featured $<45 \%$ sequence identity of the RT gene to exemplars of the other two genera [1].

Yet, it was recently shown that, similar to Hemiviruses, Sireviruses use a short 9bp segment of the ${ }^{\text {met }}$ tRNA [19]. Furthermore, ICTV only contains a small number of exemplars for each genus, while the incorporation of the vast number of new LTR-RTN species from genome sequencing projects in the classification system is understandably slow or absent. Hence, we decided to reassess the phylogenetic relationships within the Pseudoviridae family by conducting a NJ analysis of the downstream section of the pol polyprotein defined by the fifth conserved domain of the RT gene [23] to the end of the RNase H gene (Figure 1B). To better capture the diversity of the Pseudoviridae family, we used a large set of elements (Additional file 1) including all ICTV exemplars of the three genera, LTRRTNs used in previous similar studies [15,16,19], and Sirevirus representatives from all plant hosts that are present in MASiVEdb. The phylogenetic tree revealed that Sireviruses form the only monophyletic group (with 100\% bootstrap support), whilst there is no clear distinction between Hemiviruses and Pseudoviruses. Our previous analysis also showed that elements of these two genera do not differ in their genome characteristics, in contrast to the unique Sirevirus genome [19].

Consequently, we believe that further research is needed, in part through the addition of more exemplar species in ICTV, to elucidate the phylogenies of Pseudoviruses and Hemiviruses. Moreover, it may be appropriate for ICTV to include the distinctive genome structure of Sireviruses in its phylogenetic-based genus definition. Such criteria may also be used for describing other LTR-RTN genera, if future research uncovers similar findings.

\section{Conclusions}

The ICTV, and especially the TE-based system proposed by Wicker et al. [2], are indispensable resources for the challenging identification and annotation of TEs in eukaryotic genomes. However, the explosion of available sequence data and analytical tools strongly support the need for these systems to be revisited and revised: a taxonomic level is now required in the TE-based system that will make the Sirevirus and other ICTV genera available for eukaryotic genome studies. Meanwhile, the phylogenetic relationship of the Pseudovirus and Hemivirus genera should be further clarified. Finally, a similar set of analyses should also take place within the Metaviridae/ Gypsy family/superfamily. Such developments will pave the way for identifying other TE genera with unique characteristics like Sireviruses, and will encourage research that may shed light on their integrative impact on the evolution of their host genomes.

\section{Additional file}

Additional file 1: List of the RT/RNase $\mathrm{H}$ peptide sequences of the elements that were used for the construction of the Pseudoviridae phylogenetic tree.

\section{Competing interests}

The authors declare that they have no competing interests.

\section{Authors' contribution}

$A B$ conceived and coordinated the project, and drafted the manuscript. ND drafted the manuscript. All authors read and approved the final manuscript.

\section{Acknowledgements}

This work was partially supported by the Hellenic General Secretariat for Research and Technology (GSRT). ND is currently supported by CEITEC MU (CZ.1.05/1.1.00/02.0068) and project SuPReMMe (CZ.1.07/2.3.00/20.0045).

\section{Author details}

${ }^{1}$ Institute of Applied Biosciences, Centre for Research and Technology Hellas, Thessaloniki 57001, Greece. ${ }^{2}$ Central European Institute of Technology, Masaryk University, Brno, Czech Republic.

Received: 1 October 2012 Accepted: 22 January 2013

Published: 4 March 2013

\section{References}

1. King A, Adams M, Carstens E, Lefkowitz E: Virus taxonomy: classification and nomenclature of viruses: Ninth Report of the International Committee on Taxonomy of Viruses. San Diego, CA: Elsevier Academic Press; 2012.

2. Wicker T, Sabot F, Hua-Van A, Bennetzen JL, Capy P, Chalhoub B, Flavell A Leroy P, Morgante M, Panaud O, Paux E, SanMiguel P, Schulman AH: A unified classification system for eukaryotic transposable elements. Nat Rev Genet 2007, 8:973-982.

3. Tidona C, Darai G: The Springer Index of Viruses. 2nd edition. 2011. http:// www.springer.com/biomed/virology/book/978-0-387-95918-4.

4. The ITIS Catalogue of life. http://www.catalogueoflife.org/.

5. Adams MJ, Antoniw JF: DPVweb: a comprehensive database of plant and fungal virus genes and genomes. Nucleic Acids Res 2006, 34:D382-D385.

6. Pelisson A, Teysset L, Chalvet F, Kim A, Prud'homme N, Terzian C, Bucheton A: About the origin of retroviruses and the co-evolution of the gypsy retrovirus with the Drosophila flamenco host gene. Genetica 1997, 100:29-37.

7. Kim A, Terzian C, Santamaria P, Pelisson A, Prudhomme N, Bucheton A Retroviruses in invertebrates: the gypsy retrotransposon is apparently an infectious retrovirus of Drosophila melanogaster. Proc Natl Acad Sci U S A 1994, 91:1285-1289.

8. Llorens C, Munoz-Pomer A, Bernad L, Botella H, Moya A: Network dynamics of eukaryotic LTR retroelements beyond phylogenetic trees. Biol Direct 2009, 4:41.

9. Havecker ER, Gao X, Voytas DF: The diversity of LTR retrotransposons. Genome Biol 2004, 5:225.

10. Peterson-Burch BD, Voytas DF: Genes of the Pseudoviridae (Ty1/copia retrotransposons). Mol Biol Evol 2002, 19:1832-1845.

11. Laten HM, Havecker ER, Farmer LM, Voytas DF: SIRE1, an endogenous retrovirus family from Glycine max, is highly homogenous and evolutionarily young. Mol Biol Evol 2003, 20:1222-1230.

12. Laten HM, Majumdar A, Gaucher EA: SIRE-1, a copia/Ty1-like retroelement from soybean, encodes a retroviral envelope-like protein. Proc Natl Acad Sci U S A 1998, 95:6897-6902

13. Baucom RS, Estill JC, Chaparro C, Upshaw N, Jogi A, Deragon JM, Westerman RP, SanMiguel PJ, Bennetzen JL: Exceptional diversity, nonrandom distribution, and rapid evolution of retroelements in the B73 maize genome. PLOS Genet 2009, 5:e1000732.

14. Kronmiller BA, Wise RP: TEnest: automated chronological annotation and visualization of nested plant transposable elements. Plant Physiol 2008, 146:45-59.

15. Gao X, Havecker ER, Baranov PV, Atkins JF, Voytas DF: Translational recoding signals between gag and pol in diverse LTR retrotransposons. RNA 2003, 9:1422-1430. 
16. Havecker ER, Gao X, Voytas DF: The sireviruses, a plant-specific lineage of the Ty1/copia retrotransposons, interact with a family of proteins related to dynein light chain. Plant Physiol 2005, 139:857-868.

17. Holligan D, Zhang XY, Jiang N, Pritham EJ, Wessler SR: The transposable element landscape of the model legume Lotus japonicus. Genetics 2006, 174:2215-2228.

18. Weber B, Wenke T, Frommel U, Schmidt T, Heitkam T: The Ty1-copia families SALIRE and Cotzilla populating the Beta vulgaris genome show remarkable differences in abundance, chromosomal distribution, and age. Chromosome Res 2010, 18:247-263.

19. Bousios A, Darzentas N, Tsaftaris A, Pearce SR: Highly conserved motifs in non-coding regions of Sirevirus retrotransposons: the key for their pattern of distribution within and across plants? BMC Genomics 2010, 11:89.

20. Darzentas N, Bousios A, Apostolidou V, Tsaftaris AS: MASiVE: Mapping and Analysis of SireVirus Elements in plant genome sequences. Bioinformatics 2010, 26:2452-2454.

21. Bousios A, Kourmpetis YAl, Pavlidis P, Minga E, Tsaftaris A, Darzentas N: The turbulent life of Sirevirus retrotransposons and the evolution of the maize genome: more than ten thousand elements tell the story. Plant $J$ 2012, 69:475-488.

22. Bousios A, Minga E, Kalitsou N, Pantermali M, Tsaballa A, Darzentas N: MASiVEdb: the Sirevirus Plant Retrotransposon Database. BMC Genomics 2012, 13:158.

23. Xiong Y, Eickbush TH: Origin and evolution of retroelements based upon their reverse transcriptase sequences. EMBO J 1990, 9:3353-3362.

doi:10.1186/1759-8753-4-9

Cite this article as: Bousios and Darzentas: Sirevirus LTR

retrotransposons: phylogenetic misconceptions in the plant world.

Mobile DNA 2013 4:9.

\section{Submit your next manuscript to BioMed Central and take full advantage of:}

- Convenient online submission

- Thorough peer review

- No space constraints or color figure charges

- Immediate publication on acceptance

- Inclusion in PubMed, CAS, Scopus and Google Scholar

- Research which is freely available for redistribution 